\title{
Lgr5-positive cells in the lung and their clinical significance in patients with lung adenocarcinoma
}

\author{
XIAOHAN ZHANG ${ }^{1 *}$, MIAO XU ${ }^{2}$, SHIXIN SU $^{1}$, ZEQI ZHOU ${ }^{1}$, HONG YANG $^{3}$, SUWEN ZHAO $^{1}$, \\ DANNI ZENG ${ }^{1}$, KAIYING YANG ${ }^{1}$, YANAN LIU ${ }^{1}$ LIJING WANG $^{1 *}$ and JIANGCHAO LI ${ }^{1}$ \\ ${ }^{1}$ Vascular Biology Research Institute, Guangdong Pharmaceutical University, Guangzhou, Guangdong 510006; \\ ${ }^{2}$ State Key Laboratory of Oncology in South China and ${ }^{3}$ Department of Thoracic Surgery, Sun Yat-Sen \\ University Cancer Center, Guangzhou, Guangdong 510060, P.R. China
}

Received December 15, 2015; Accepted May 20, 2016

DOI: $10.3892 / \mathrm{mco} .2016 .934$

\begin{abstract}
Leucine-rich repeat-containing G-protein coupled receptor 5 (Lgr5) is well-characterized as a marker of intestinal stem cells and certain types of tumor stem cells, where positive cells may develop into intestinal epithelial cells or intestinal adenomas. However, the roles of Lgr5 in the lung and in lung tumors remain unclear. An immunohistochemistry (IHC) analysis was performed to detect the expression of Lgr5 in the lung from a normal mouse. Histopathological sections of the lungs from Lgr5 heterozygous knockout mice $\left(\mathrm{Lgr}^{+/}\right)$were observed following with hematoxylin and eosin. Furthermore, tissue microarrays containing tumor cores from lung cancer patients were also analyzed by IHC. Lgr5-positive cells were present in the pulmonary alveoli and bronchi of normal mice, whereas the lungs of $\mathrm{Lgr}^{+/-}$mice lost their normal morphological structure compared with the lungs of the normal mice. Lgr5 was expressed in lung adenocarcinoma, however, not in squamous carcinoma, and Lgr5 expression was positively associated with tumor, node, metastasis stage. Lgr5 is expressed in normal murine lung and is associated with TNM stage in patients with lung adenocarcinoma.
\end{abstract}

\section{Introduction}

In 1998, Hsu et al (1) found that the leucine-rich repeat-containing G-protein coupled receptor 5 (Lgr5) was homologous to the gonadotropin and thyrotropin receptors in Drosophila (1). Lgr5 is expressed in the eye, brain, mammary glands, hair follicles, stomach, intestines and other tissues $(2,3)$. Lgr5 serves an important role in the Wnt signaling pathway, which is intimately involved in tumor

Correspondence to: Professor Jiangchao Li or Dr Lijing Wang, Vascular Biology Research Institute, Guangdong Pharmaceutical University, 280 Wai Huan Dong Road, Guangzhou Higher Education Mega Center, Guangzhou, Guangdong 510006, P.R. China

E-mail: lijiangchao1234@163.com

E-mail: wanglijing62@163.com

Key words: Lgr5, lung cancer, transgenic mice, TNM stage development (4-6). Lgr5 is a marker of stem cells in the intestine, mammary glands and hair follicles. Lgr5 is also expressed in intestinal tumors $(7,8)$, which are composed of crypt base columnar cells and stem cells. In addition, Lgr5 is expressed in hepatocellular carcinoma, breast cancer and gastric carcinoma. However, its role in normal lung and in lung cancer remains unknown.

Lung cancer is the leading cause of cancer-assocaited mortality among males in developed countries (9). Few studies reporting on Lgr5 in lung cancer have been published in recent years $(10,11)$. To investigate whether Lgr5 serves a role in lung cancer similar to that in colorectal cancer and to determine whether Lgr 5 functions in the maintenance of lung structure, the present study assessed the structure of the lungs in Lgr5 heterozygous knockout mice $\left(\mathrm{Lgr}^{+/}\right)$. Notbaly, it was demonstrated that the lungs of $\mathrm{Lgr}^{+/}$mice were abnormal compared with those of wild-type (WT) mice, which suggested that Lgr5 functions in the maintenance of normal lung structure. Furthermore, Lgr5 expression was analyzed in tumor tissues of patients with lung cancer. The present study indicated that Lgr5 serves an important role in lung adenocarcinoma and provided some meaningful insight into the presence of Lgr5-positive cells in the lungs and Lgr5 expression in lung cancer.

\section{Materials and methods}

Mice. Genetically engineered mice [B6.129P2-Lgr5tm1 (cre/ERT2) Cle/J] were donated to our laboratory by Professor Geng (Department of Biologic and Materials Sciences, University of Michigan School of Dentistry, Ann Arbor, MI, USA). This mouse model was produced by knock-in of the Lgr5-EGFP-IRES-creERT2 allele, which results in the loss of function of one Lgr5 allele and the expression of the CreERT2-EGFP fusion protein. Lgr5 mice do not exhibit any obvious abnormalities in terms of birth rate or any abnormal physiological properties. By contrast, the homozygous deletion of Lrg5 in mice resulted in neonatal lethality (12). To investigate the role of Lgr5 in the lungs, $\mathrm{Lgr5}^{+/-}$mice were used in the present study. The mice were housed in an individual ventilated cage (IVC) environment at $24 \pm 2^{\circ} \mathrm{C}$ and $60 \pm 5 \%$ humidity with $12 \mathrm{~h}$ light/dark cycles. The mice were fed a bacteria-free diet. All mice were sacrificed under diethyl ether anesthesia. Guangdong 
College of Pharmacy Approved Animal Ethics Committee approved all murine experiments. To identify the genotype of the mice, DNA was extracted from a $0.5-1 \mathrm{~cm}$ portion of the tail. The DNA was amplified in polymerase chain reaction (PCR) amplification buffer (2X PCR mix; Promega, Beijing, China), and the PCR products were subsequently run on a $2 \%$ agarose gel. The PCR product of the homozygous mice was $174 \mathrm{bp}$, the heterozygous mice PCR product was $174 \mathrm{bp}$ and the WT mice product was $298 \mathrm{bp}$. The primers used were as follows: Common (sequence no. 8060), 5'-CTG CTC TCT GCT CCC AGT CT-3'; WT reverse (sequence no. 8061), 5'-ATA CCC CAT CCC TTT TGA GC-3'; mutant reverse (oIMR9402) 5'-GAA CTT CAG GGT CAG CTT GC-3'. The PCR system and PCR reaction conditions were in accordance with the protocols of the Jackson Laboratory (Bar Harbor, ME, USA).

Collection of tissue specimens. Lgr5 expression was evaluated in a total of 42 primary non-small cell lung cancers (NSCLCs) and 28 matched normal adjacent lung tissue samples by immunohistochemistry (IHC). The 42 samples included 22 lung adenocarcinoma samples and 20 squamous cell carcinoma samples. In addition, a tissue microarray (TMA) that contained 80 cases of lung adenocarcinoma was also included. Therefore, the total number of lung adenocarcinoma cases was 102 , whereas the total number of squamous cell carcinoma samples was 20 . The clinical features of the patients with NSCLC are outlined in Table I. All specimens were collected from patients who were admitted to the Department of Thoracic Surgery, Sun Yat-Sen University Cancer Center, (Guangzhou, China) between October 9, 2009 and July 12, 2014 with patient consent and institutional review board approval. All patients signed informed consent for the use of their clinical specimens for medical research. The samples in this study were approved by the Committee for Ethical Review of Research at Sun Yat-Sen University.

Histology. The paraffin embedded tissues were sectioned at a thickness of 3-5 $\mu \mathrm{m}$, and hematoxylin and eosin (H\&E) staining was performed, as previously described (13). Briefly, IHC was performed as follows: Antigen retrieval with ethylenediaminetetraacetic acid solution ( $\mathrm{pH}$ 9.0), incubation of the slides in $3 \% \mathrm{H}_{2} \mathrm{O}_{2}$ at room temperature for $12 \mathrm{~min}$, followed by washes and the blocking of non-specific proteins. The antibody was applied to the slides, which were subsequently incubated at $4^{\circ} \mathrm{C}$ overnight. The tissue sections were washed again and the secondary antibody was applied to the slides. Finally, diaminobenzidine was used to visualize the positive staining on the slides. The primary Lgr5 antibody was used at a dilution of 1:100 (cat. no. sc-135238; Santa Cruz Biotechnology, Inc., Santa Cruz, CA, USA). The anti-rabbit secondary antibody was conjugated to horseradish peroxidase and was used at a dilution of 1:500 (cat. no. GK500705). The diaminobenzidine chromogen system was purchased from Gene Tech Co., Ltd. (Shanghai, China). The slides were viewed on a BX51 Olympus microscope (Olympus Corporation, Shibuya, Japan).

Statistical analysis. All statistical analyses were performed using GraphPad Prism software version 5 (Graphpad Software, CA, USA). The $\chi^{2}$ test or Fisher's exact test was used to analyze the association of Lgr5 expression with the clinicopatho- logical parameters of the patients. The survival curves were plotted according to the Kaplan-Meier method to evaluate the Lgr5 expression levels with respect to the survival rate. The multivariate survival analyses were performed by a Cox proportional hazard model using the Wald test. $\mathrm{P}<0.05$ was considered to indicate a statistically significant difference..

\section{Results}

Lgr5-positive cells are present in mouse lung tissue. Lgr5 is well-characterized as a marker of normal stem cells in the intestine and as a marker of cancer stem cells. The Lgr5 homozygous knockout in mice resulted in neonatal lethality (12). The present study suggested that Lgr5 serves an important role in development. However, the role of Lgr5-positive cells in the lung remains unknown. To investigate whether cells in normal mouse lung express Lgr5, normal lung tissue was obtained from C57/BL WT mice and the expression of Lgr5 was determined by IHC using a specific antibody. To confirm that the antibody was specific, normal mouse intestine was stained. The results are shown in Fig. 1A. In the lung tissue, the results demonstrated that some Lgr5-positive cells were present in the bronchi and alveoli (Fig. 1B and C). The present study next determined whether the knockout of Lgr5 affected lung structure and found that the structure of the lung tissue of Lgr5 heterozygous knockout mice ( $\operatorname{Lgr} 5^{+/-}$mice) differed from that of the WT mice. This finding indicated that the lung septa of $\operatorname{Lgr} 5^{+-}$mice were thicker and that the lung structure was irregular compared with the WT mice (Fig. 1E).

Lgr5 is expressed in lung adenocarcinoma, but not in squamous carcinoma. Based on the above IHC and histology results, the present study hypothesized that Lgr5 may serve a function in the maintenance of the morphology of bronchi or alveoli in the normal lung. It must be considered that lung tumor initiating cells are partly derived from the bronchi and that Lgr5 is a well-known marker of intestinal cancer stem cells. Therefore, in lung cancer, the present study assumed that Lgr5 is associated with tumor formation or to the tumor, node, metastasis (TNM) stage. Next, 122 lung cancer cases, including 102 cases of adenocarcinoma and 20 cases of squamous carcinoma, were subjected to IHC. The results showed that Lgr5 is not expressed in squamous cell carcinoma $(0 / 20$; P<0.001; Table I; Fig. 2A), however, is expressed in adenocarcinoma (47/122). The representative image of the staining is presented in Fig. 2B and C. Notably, in certain cases, Lgr5-positive cells were present in areas of tumor necrosis (Fig. 2D), areas adjacent to the tumor (Fig. 2E), and the lymphatic spaces and vascular lumina (Fig. 2F).

Survival analysis of patients with lung adenocarcinoma as a function of Lgr5 expression. The above data showed that Lgr5 is expressed in lung adenocarcinoma, but not in squamous carcinoma. Furthermore, the present study stained one tissue microarray that included 60 cases of lung adenocarcinoma to assess its expression by IHC using an Lgr5 antibody. These cases were classified into two groups as either ' + ' or '-', as shown in the representative image in Fig. 3A. The positive group was indicated by ' + ' and the negative group was indicated by '-'. 
Table I. Correlation between Lgr5 expression and the clinical and pathological characteristics of 122 cases of primary non-small cell lung cancer.

\begin{tabular}{|c|c|c|c|c|}
\hline \multirow[b]{2}{*}{ Clinical feature } & \multirow[b]{2}{*}{ No. patients } & \multicolumn{2}{|c|}{ Lgr5 expression (\%) } & \multirow[b]{2}{*}{ P-value } \\
\hline & & Negative $(\%)$ & Positive (\%) & \\
\hline Gender & & & & 0.269 \\
\hline Male & 75 & $49(65.3)$ & $26(34.7)$ & \\
\hline Female & 47 & $26(55.3)$ & $21(44.7)$ & \\
\hline Age & & & & 0.421 \\
\hline$\leq 60$-years-old & 68 & $46(67.6)$ & $22(32.4)$ & \\
\hline$>60$-years-old & 64 & $39(60.9)$ & $25(39.1)$ & \\
\hline Tumor size ${ }^{\mathrm{a}, \mathrm{b}}$ & & & & 0.075 \\
\hline$\leq 5 \mathrm{~cm}^{3}$ & 59 & $31(52.5)$ & $27(47.5)$ & \\
\hline$>5 \mathrm{~cm}^{3}$ & 63 & $43(84.10)$ & $20(15.9)$ & \\
\hline Tumor invasion $^{\mathrm{c}}$ & & & & 0.351 \\
\hline $\mathrm{T} 1$ & 12 & $6(50.0)$ & $6(50.0)$ & \\
\hline $\mathrm{T} 2$ & 65 & $28(43.1)$ & $37(56.9)$ & \\
\hline $\mathrm{T} 3$ & 45 & $27(60.0)$ & $18(40.0)$ & \\
\hline Lymph node metastasis & & & & 0.569 \\
\hline No & 82 & $52(63.4)$ & $30(36.6)$ & \\
\hline N1 & 40 & $23(57.5)$ & $17(42.5)$ & \\
\hline $\mathrm{AD}$ and $\mathrm{SS}$ & & & & $<0.001$ \\
\hline Adenocarcinoma & 102 & $55(53.9)$ & $47(46.1)$ & \\
\hline Squamous & 20 & $20(100)$ & $0(0.0)$ & \\
\hline TNM stage ${ }^{\mathrm{d}}$ & & & & 0.026 \\
\hline I-II & 81 & $56(69.1)$ & $25(30.9)$ & \\
\hline III-IV & 41 & $19(46.3)$ & $22(53.7)$ & \\
\hline
\end{tabular}

${ }^{a}$ Tumor size was measured and obtained by the formula $0.5 \mathrm{x}$ length $\mathrm{x}$ (width) ${ }^{2}$. ${ }^{\mathrm{b}}$ Partial data are not available and all statistics were based on the

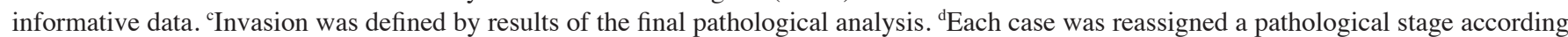
to the $7^{\text {th }}$ edition of the TNM classification. AD, adenocarcinoma; SS, squamous carcinoma; TNM, tumor node metastasis; Lgr5, Leucine-rich repeat-containing G-protein coupled receptor 5.

A survival analysis was performed for all patients with lung adenocarcinoma (paraffin blocks, 22 cases; TMA, 80 cases) using the Kaplan-Meier method. The follow-up periods ranged between 4 and 157 months. To evaluate the association between Lgr5 expression in lung adenocarcinoma cells and the clinicopathological parameters, the data from the patients was summarized in Table I. Lgr5 expression was not associated with tumor size $(\mathrm{P}=0.075)$. In addition, no significant association was observed between Lgr5 expression and age, gender, tumor invasion or lymph node metastasis. However, Lgr5 expression was associated with TNM stage $(\mathrm{P}=0.026)$.

Additionally, the median survival was 30.5 months for patients in the Lgr5-negative group $(n=55)$ and 12 months $(n=47)$ for patients in the Lgr5-positive group, which indicated a significantly poorer survival rate of the Lgr5-positive group compared with the Lgr5-negative group ( $\mathrm{P}=0.033)$. Next, univariable and multivariable analyses were performed to assess the effect of Lgr5 expression on survival. A Cox proportional hazards regression model was subsequently applied and the effect of Lgr5 on survival was estimated. The crude hazard ratio (HR) of the Lgr5-positive tumors compared with the Lgr5-negative tumors was 1.618 [95\% confidence interval $(\mathrm{CI}), 1.06-2.48 ; \mathrm{P}=0.027]$. To estimate the independent pro-diagnostic effect of Lgr5 on survival, the present study adjusted for confounding factors. The present study involved 59 lung adenocarcinoma patients with lung cancer-associated mortalities and 5 variables that were included in the multivariate regression model. To determine whether Lgr5 expression was a single variable and not a potential confounding factor, a propensity score was applied. The five variables were clinicopathological factors, including age, tumor size, TNM stage, tumor invasion and metastasis. The adjusted HR of the Lgr5-positive group was 0.396 (95\% CI, -0.28-1.06; $\mathrm{P}=0.15$ ) compared with the Lgr5-negative group, which suggested that Lgr5 expression is not an independent risk factor for poorer survival (Table II).

\section{Discussion}

Lgr5 is regarded as a marker of intestinal stem cells, which has attracted a significant amount of attention to this protein (8,14-19). However, its role in the lungs and in lung cancer remains unclear (10). The present study demonstrated 
A Small intestine

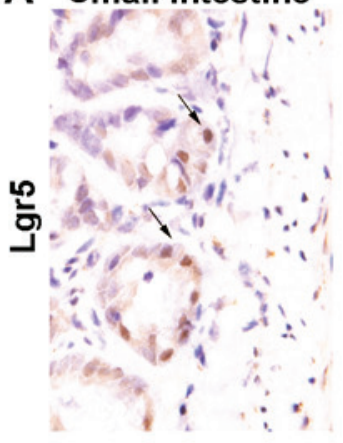

D

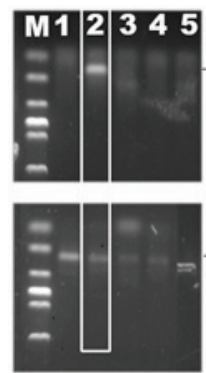

B

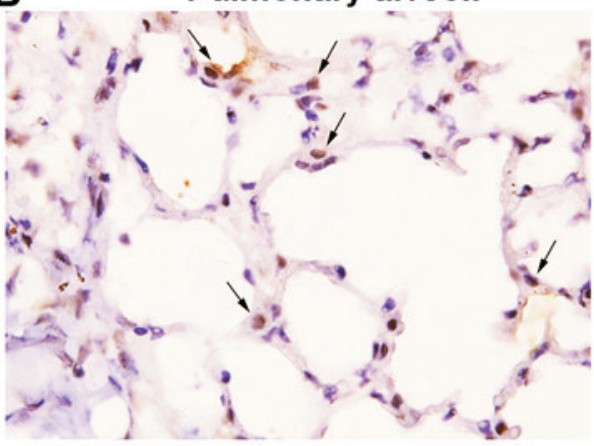

Pulmonary alveoli

E

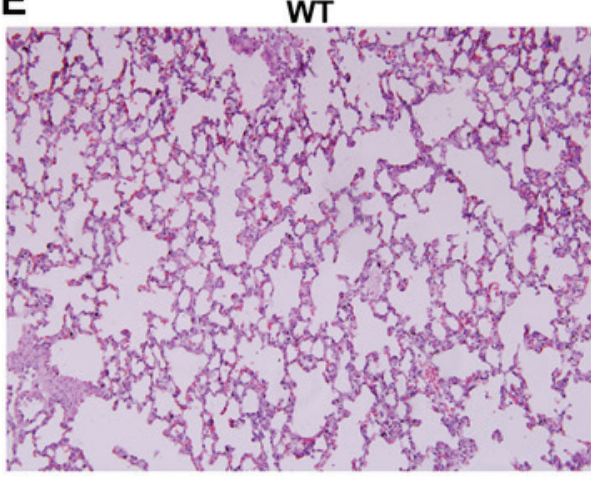

C
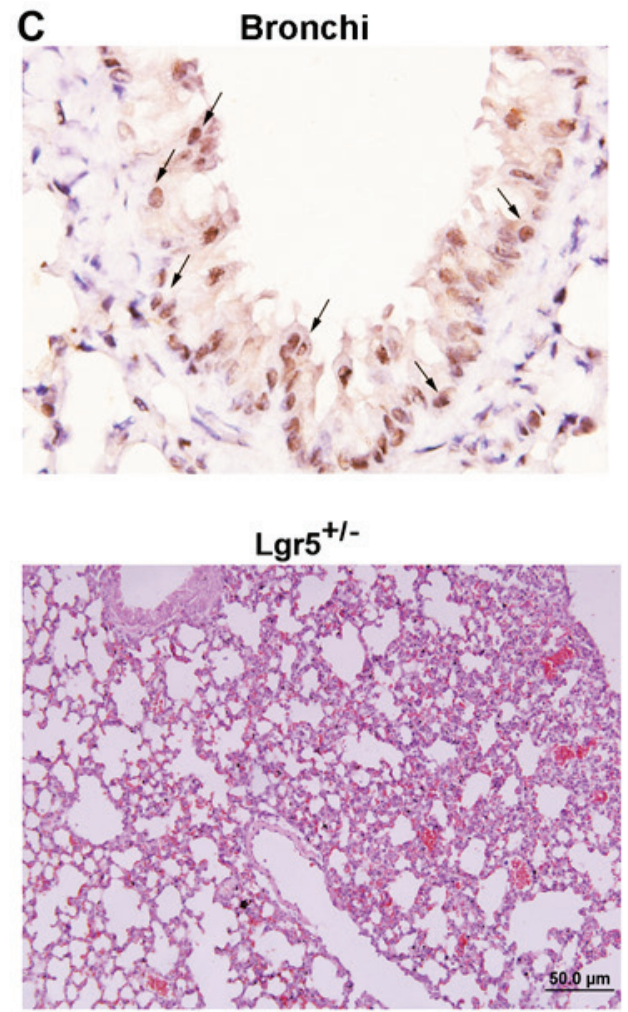

Figure 1. Lgr5 expression in the mouse lung. (A) The sensitivity of the Lgr5 antibody was confirmed by staining intestinal tissue of WT mice. Lgr5-positive cells are indicated by arrows. (B) A few Lgr5-positive cells are present in the alveoli of mouse lung, as indicated by arrows (magnification, $\mathrm{x} 1,000$ ). (C) A few Lgr5-positive cells were present in the lung bronchi (magnification, $\mathrm{x} 1,000$ ), as indicated by arrows. (D) Identification of Lgr $5^{+/-}$mice was performed by genotyping. Bands of 298 bp and 174 bp were obtained by polymerase chain reaction amplification and are indicative of the Lgr $5^{+/-}$genotype. Lanes 1 and 3-5 are WT mice and lane 2 is the Lgr5 $5^{+/}$mouse. (E) Representative hematoxylin and eosin staining of Lgr5 $5^{+/}$mouse lung and WT mouse lung tissue. Interlobular septa of Lgr5 ${ }^{+/-}$mouse lung are thickened and abnormal hyperplasia in the bronchi and light pulmonary congestion were also observed (magnification, $\mathrm{x} 200$ ). WT, wild-type; Lgr5, Leucine-rich repeat-containing G-protein coupled receptor 5; bp, base pairs; M, marker.
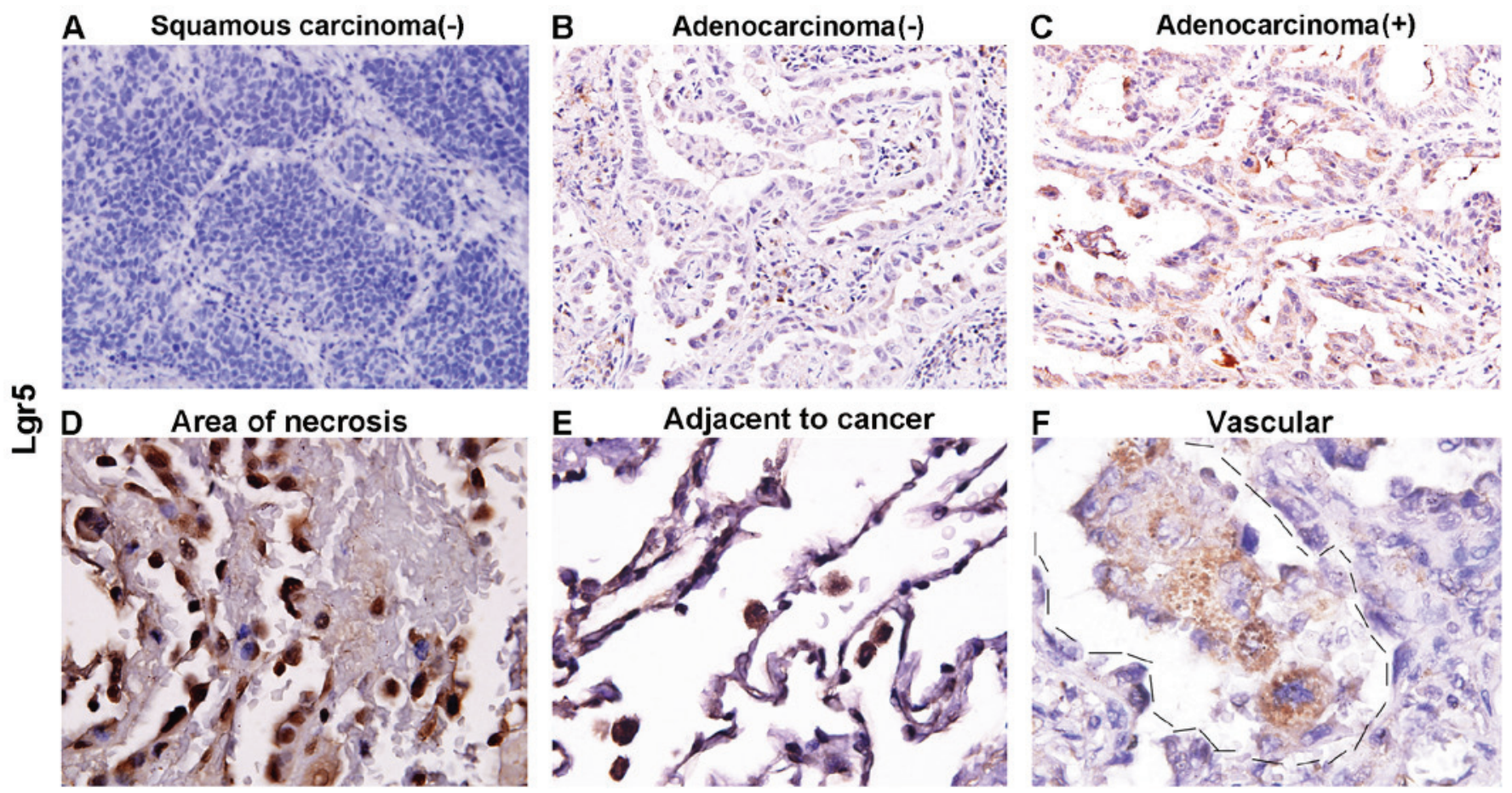

Figure 2. Lgr5 is expressed in lung adenocarcinoma and is not expressed in squamous carcinoma. (A) A representative image of Lgr5 negativity in squamous carcinoma (magnification, x400). (B) A representative image of lung adenocarcinoma indicates that certain samples did not express Lgr5 (magnification, x400). (C) Certain lung adenocarcinoma tissues expressed Lgr5 (magnification, x400). (D) Lgr5-positive cells are present in the necrotic area of the tumor tissues (magnification, x1,000). (E) Lgr5-positive cells also appear in regions adjacent to the carcinoma (magnification, x400). (F) Lgr5-positive cells in the lumen (magnification, x1,000). Lgr5, Leucine-rich repeat-containing G-protein coupled receptor 5. 
Table II. Univariate and multivariate analyses of the overall survival rate and the clinicopathological parameters of 122 patients with lung cancer using the Cox proportional hazards regression model.

\begin{tabular}{|c|c|c|c|}
\hline \multirow[b]{2}{*}{ Factors } & \multirow{2}{*}{$\frac{\text { Univariate }^{\mathrm{a}}}{\text { P-value }}$} & \multicolumn{2}{|c|}{ Multivariate $^{\mathrm{b}}$} \\
\hline & & Hazard ratio $(95 \% \mathrm{CI})$ & P-value \\
\hline $\begin{array}{l}\text { Age } \\
\leq 60 \text {-years-old } \\
>60 \text {-years-old }\end{array}$ & 0.136 & $1.32(0.944-2.435)$ & 0.094 \\
\hline $\begin{array}{l}\text { Tumor size } \\
\leq 10 \mathrm{~cm}^{3} \\
>10 \mathrm{~cm}^{3}\end{array}$ & 0.032 & $1.12(0.832-1.482)$ & 0.696 \\
\hline $\begin{array}{l}\text { Tumor invasion } \\
\text { T1 } \\
\text { T2 } \\
\text { T3 }\end{array}$ & 0.033 & $1.58(0.951-2.810)$ & 0.173 \\
\hline $\begin{array}{l}\text { Lymph node metastasis } \\
\text { N0 } \\
\text { N1 }\end{array}$ & 0.001 & $1.812(0.611-4.650)$ & 0.308 \\
\hline $\begin{array}{l}\text { TNM stage } \\
\text { I-II } \\
\text { III-IV }\end{array}$ & 0.005 & $0.861(0.470-2.667)$ & 0.897 \\
\hline $\begin{array}{l}\text { Lgr5 } \\
\text { Negative } \\
\text { Positive }\end{array}$ & 0.076 & $3.36(1.24-10.63)$ & 0.036 \\
\hline
\end{tabular}

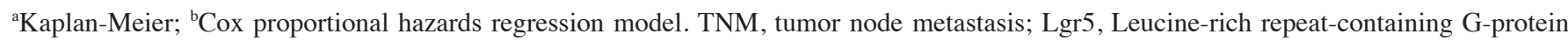
coupled receptor 5 .

for the first time, to the best of our knowledge, that the lungs of Lgr5 heterozygous mice exhibited structural abnormalities. Specifically, the pulmonary interstitium was thickened according to H\&E staining. Additionally, Lgr5-positive cells were found in the mouse lung, including the alveoli. In addition, a few cells expressing Lgr5 were also found in the basal layer of the bronchial tissue. The above results suggested that Lgr5 may serve a significant role in the lung bronchi and alveoli. However, a determination of the function of these positive cells requires further clarification.

In both the human and mouse gut, Lgr5-positive cells located in the intestinal crypts protect Paneth cells and are regarded as tumor stem cells (18). In the present study, it was revealed that certain cells in normal lung tissue express Lgr5. This finding led us to question whether Lgr5 serves a role in normal lung tissue or if it is associated with lung cancer. Certain lung cancer cells are derived from the lung bronchi; therefore, Lgr5 expression in lung tumor tissues from patients with lung cancer was examined. Notably, Lgr5 is only expressed in lung adenocarcinoma and its expression is associated with TNM stage; however, Lgr5 expression not an independent factor for survival. In another study, the Lgr5-positive rate in lung adenocarcinoma was $\sim 1 / 10$ (11). However, in the present study, the rate reached $\sim 50 \%$. However, as the reasons that underlie this difference are unknown, additional studies are required for clarification.

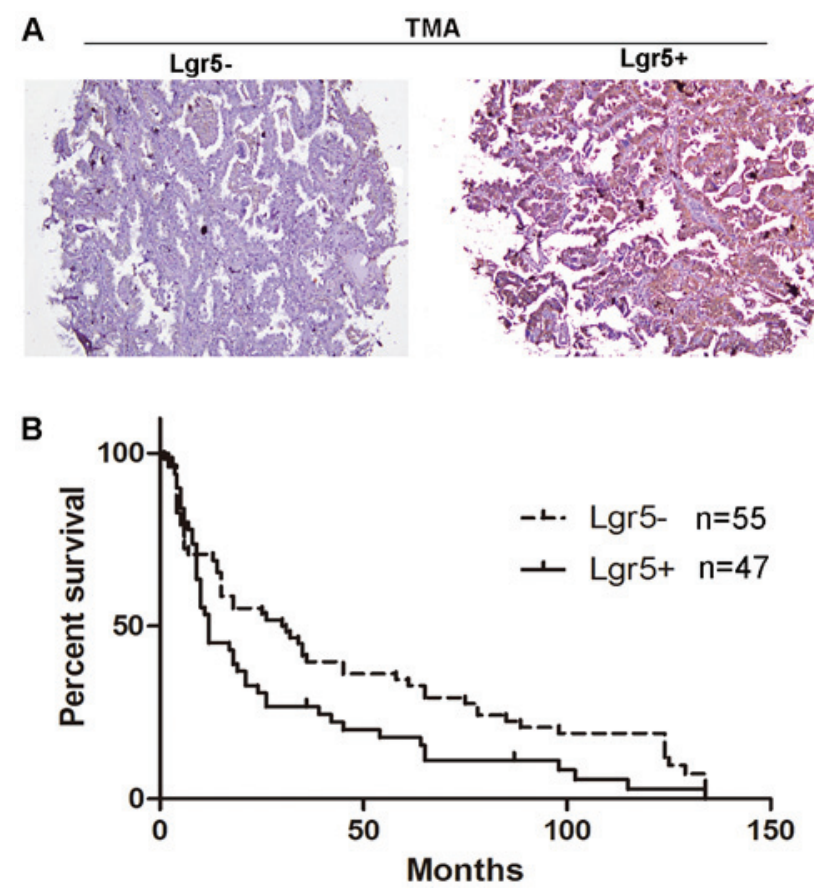

Figure 3. Survival analysis. (A) A representative image of the TMA immunohistochemistry results demonstrating negative (left) and positive (right) Lgr5 staining. (B) Kaplan-Meier analysis revealed that Lgr5 expression was significantly associated with poor overall survival of patients with lung adenocarcinoma $(\mathrm{P}=0.019)$. Lgr5, Leucine-rich repeat-containing G-protein coupled receptor 5; TMA, tissue microarray. 
In previous studies, researchers have demonstrated that Lgr5 expression is associated with colorectal tumorigenesis and even colorectal cancer recurrence (20). Previous studies have also reported that Lgr5 expression is correlated with tumor invasion and metastasis $(15,21,22)$. However, in the present experiments, the data did not indicate this phenomenon in lung adenocarcinoma. This difference may be because Lgr5 serves different roles in lung tumors compared with intestinal tumors. Notably, the presence of Lgr5-positive cells in lymphatic vessels were observed. Therefore, more experiments are required to investigate whether Lgr5 expression is associated with tumor metastasis (Fig. 3C).

In conclusion, the present study provided novel insights into the role of Lgr5 in normal lung tissue and lung cancer. It will be conducive for us to learn the role and function of Lgr5 in lung cancer.

\section{Acknowledgements}

The authors would like to thank the staff at the Department of Pathology, Sun Yat-Sen University Cancer Center (Guangzhou, China) for support in the collection of the clinical samples. The present study was supported by the National Natural Science Foundation of China (nos. 81472336 and 31471290), the academic and professional development funds of the Guangdong Provincial Department of Education (no. 2013KJCX0108) and the non-profit foundation of Guangdong Province in China (nos. 2014A020212313 and 2015A030302086).

\section{References}

1. Hsu SY, Liang SG and Hsueh AJ: Characterization of two LGR genes homologous to gonadotropin and thyrotropin receptors with extracellular leucine-rich repeats and a $\mathrm{G}$ protein-coupled, seven-transmembrane region. Mol Endocrinol 12: 1830-1845, 1998.

2. Sukhdeo K, Koch CE, Miller TE, Zhou H, Rivera M, Yan K, Cepko CL, Lathia JD and Rich JN: The Lgr5 transgene is expressed specifically in glycinergic amacrine cells in the mouse retina. Exp Eye Res 119: 106-110, 2014.

3. Vroegindeweij E, van Mourik I, Cupedo T and Cornelissen JJ: Characterization of Lgr5-positive epithelial cells in the murine thymus. Eur J Immunol 43: 1243-1251, 2013.

4. de Lau W, Barker N, Low TY, Koo BK, Li VS, Teunissen H, Kujala P, Haegebarth A, Peters PJ, van de Wetering M, et al: Lgr5 homologues associate with Wnt receptors and mediate R-spondin signalling. Nature 476: 293-297, 2011.

5. Huch M, Dorrell C, Boj SF, van Es JH, Li VS, van de Wetering M, Sato T, Hamer K, Sasaki N, Finegold MJ, et al: In vitro expansion of single Lgr5+ liver stem cells induced by Wnt-driven regeneration. Nature 494: 247-250, 2013.
6. Flanagan DJ, Phesse TJ, Barker N, Schwab RH, Amin N, Malaterre J, Stange DE, Nowell CJ, Currie SA, Saw JT, et al: Frizzled7 functions as a Wnt receptor in intestinal epithelial Lgr5(+) stem cells. Stem Cell Reports 4: 759-767, 2015.

7. Barker N, van Es JH, Kuipers J, Kujala P, van den Born M, Cozijnsen M, Haegebarth A, Korving J, Begthel H, Peters PJ, et al: Identification of stem cells in small intestine and colon by marker gene Lgr5. Nature 449: 1003-1007, 2007.

8. Snippert HJ, van der Flier LG, Sato T, van Es JH, van den Born M, Kroon-Veenboer C, Barker N, Klein AM, van Rheenen J, Simons BD and Clevers H: Intestinal crypt homeostasis results from neutral competition between symmetrically dividing Lgr5 stem cells. Cell 143: 134-144, 2010.

9. Torre LA, Bray F, Siegel RL, Ferlay J, Lortet-Tieulent J and Jemal A: Global cancer statistics, 2012. CA Cancer J Clin 65: 87-108, 2015.

10. Gao F, Zhou B, Xu JC, Gao X, Li SX, Zhu GC, Zhang XG and Yang C: The role of LGR5 and ALDH1A1 in non-small cell lung cancer: Cancer progression and prognosis. Biochem Biophys Res Commun 462: 91-98, 2015.

11. Ryuge S, Sato Y, Jiang SX, Wang G, Kobayashi M, Nagashio R, Katono K, Iyoda A, Satoh Y and Masuda N: The clinicopathological significance of Lgr5 expression in lung adenocarcinoma. Lung Cancer 82: 143-148, 2013.

12. Morita H, Mazerbourg S, Bouley DM, Luo CW, Kawamura K, Kuwabara Y, Baribault H, Tian $\mathrm{H}$ and Hsueh AJ: Neonatal lethality of LGR5 null mice is associated with ankyloglossia and gastrointestinal distension. Mol Cell Biol 24: 9736-9743, 2004.

13. Feldman AT and Wolfe D: Tissue processing and hematoxylin and eosin staining. Methods Mol Biol 1180: 31-43, 2014.

14. Takeda K, Kinoshita I, Shimizu Y, Matsuno Y, Shichinohe T and Dosaka-Akita H: Expression of LGR5, an intestinal stem cell marker, during each stage of colorectal tumorigenesis. Anticancer Res 31: 263-270, 2011.

15. He S, Zhou H, Zhu X, Hu S, Fei M, Wan D, Gu W, Yang X, Shi D, Zhou J, et al: Expression of Lgr5, a marker of intestinal stem cells, in colorectal cancer and its clinicopathological significance. Biomed Pharmacother 68: 507-513, 2014.

16. Metcalfe C, Kljavin NM, Ybarra R and de Sauvage FJ: Lgr5+ stem cells are indispensable for radiation-induced intestinal regeneration. Cell Stem Cell 14: 149-159, 2014.

17. Buczacki SJ, Zecchini HI, Nicholson AM, Russell R, Vermeulen L, Kemp R and Winton DJ: Intestinal label-retaining cells are secretory precursors expressing Lgr5. Nature 495: 65-69, 2013.

18. Sato T, van Es JH, Snippert HJ, Stange DE, Vries RG, van den Born M, Barker N, Shroyer NF, van de Wetering M and Clevers H: Paneth cells constitute the niche for Lgr5 stem cells in intestinal crypts. Nature 469: 415-418, 2011.

19. Becker L, Huang Q and Mashimo H: Immunostaining of Lgr5, an intestinal stem cell marker, in normal and premalignant human gastrointestinal tissue. ScientificWorldJournal 8: 1168-1176, 2008.

20. Tsuji S, Kawasaki Y, Furukawa S, Taniue K, Hayashi T, Okuno M, Hiyoshi M, Kitayama J and Akiyama T: The miR-363-GATA6-Lgr5 pathway is critical for colorectal tumourigenesis. Nat Commun 5: 3150, 2014.

21. Kleist B, Xu L, Li G and Kersten C: Expression of the adult intestinal stem cell marker Lgr5 in the metastatic cascade of colorectal cancer. Int J Clin Exp Pathol 4: 327-335, 2011.

22. Wang Y, Jiang CQ and Fan LF: Correlation of Musashi-1, Lgr5, and pEGFR expressions in human small intestinal adenocarcinomas. Tumour Biol 36: 6075-6082, 2015. 